\title{
Periureteric Mass in a Treated Case of Papillary Carcinoma of Thyroid: A Diagnostic Dilemma
}

\author{
${ }^{1}$ Department of Radiodiagnosis, Tata Memorial Hospital, Mumbai, \\ Maharashtra, India \\ 2Department of Nuclear Medicine, Tata Memorial Hospital, Mumbai, \\ Maharashtra, India \\ ${ }^{3}$ Department of Pathology, Tata Memorial Hospital, Mumbai, \\ Maharashtra, India \\ ${ }^{4}$ Department of Surgical Oncology, Tata Memorial Hospital, \\ Mumbai, Maharashtra, India
}

Sushil Panbude ${ }^{1} \quad$ Archi Agrawal $^{2} \quad$ Shubhada Kane $^{3} \quad$ Anil D’Cruz $^{4} \quad$ Supreeta Arya ${ }^{1}$

\begin{abstract}
Address for correspondence Supreeta Arya, MD, DMRD, DNB (Radio-diagnosis), Department of Radiodiagnosis, Tata Memorial Hospital, Mumbai 400012, Maharashtra, India (e-mail: supreeta.arya@gmail.com).
\end{abstract}

J Gastrointestinal Abdominal Radiol ISGAR 2019;2:58-63

\section{Case History}

A 41-year-old woman, a known case of papillary carcinoma of the thyroid, post total thyroidectomy was on regular surveillance at a tertiary referral oncology center with ultrasonography (US) of the neck and serum thyroglobulin assessment for 4 years. Histopathology report revealed classic type of differentiated papillary carcinoma of the left lobe of the thyroid with uninvolved regional nodes. Maximum diameter of tumor was $1.1 \mathrm{~cm}$. Lymphovascular emboli and perineurial invasion were absent. Extrathyroidal extension was not seen.

Four months after the 4-year follow-up visit revealed no evidence of locoregional recurrence, the patient suddenly presented with urinary frequency, left lower abdominal pain, anorexia, and weakness. Incidentally, she had a history of Koch's abdomen 8 years before for which she had received treatment. A US of the abdomen and pelvis was performed and revealed mild left hydronephrosis and an ill-defined small mass in the left hemipelvis. No other abnormality was evident. A multidetector computed tomography (MDCT) study of the abdomen and pelvis was performed on Lightspeed 16 slice, GE machine. Contrast-enhanced CT scan was acquired in venous phase with $90 \mathrm{cc}$ of iodinated non-ionic contrast (iopamidol) injected at a rate of $2.5 \mathrm{~mL} / \mathrm{s}$. Images were acquired with $5 \mathrm{~mm}$ thickness, and sagittal and coronal reconstructions were performed using retro-reconstructed $1.25 \mathrm{~mm}$ thick slices. It showed mild left hydronephrosis due to narrowing of an approximately $2-\mathrm{cm}$-long segment of lower third of the left ureter due to compression by an extrinsic mass located medial to the ureter ( $\boldsymbol{- \text { Fig. }} \mathbf{1}$ ). The mass measured $3.0 \times 2.1 \mathrm{~cm}$ and showed ill-defined margins with adjacent fat stranding. The mass extended into the region between the left external and internal iliac veins, but the vessels were free. It appeared separate from the left ovary and uterus. It showed progressive enhancement on venous and delayed received

September 2, 2018 accepted after revision

October 28, 2018
License terms

() (1) $\ominus \circledast$ 


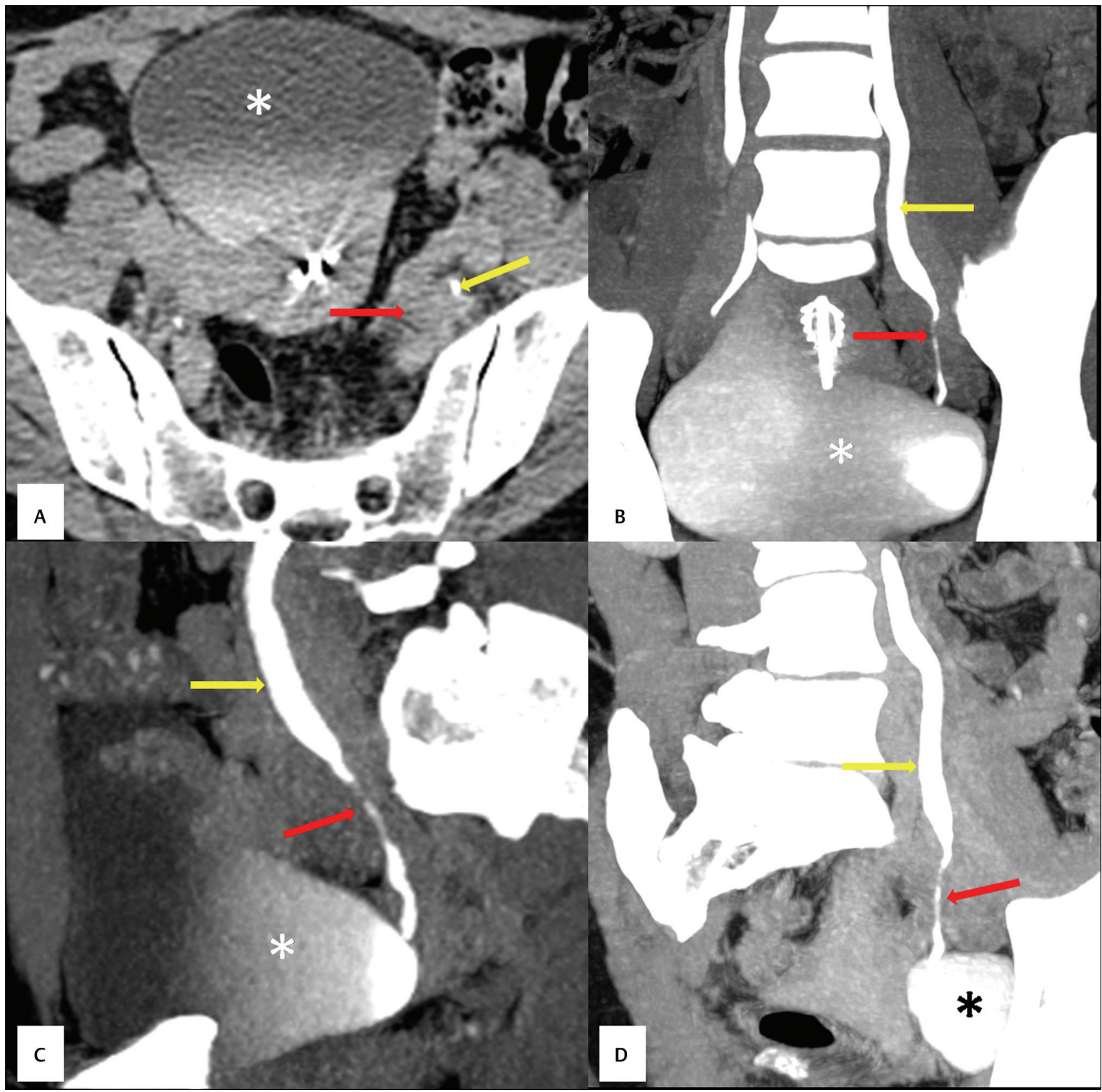

Fig. 1 Axial (A), coronal (B), sagittal (C), and oblique (D) reformatted multidetector computed tomography (MDCT) images in maximum-intensity projection in the delayed phase showing soft tissue density mass medial to the left distal ureter causing extrinsic compression of the left ureter (red arrow) near the left sacroiliac joint (SI) joint with upstream dilatation of the ureter (yellow arrow). Asterisk $\left({ }^{*}\right)$ represents urinary bladder.

postcontrast images. The ureter proximal to the mass showed dilatation, and the entire proximal ureter showed mucosal enhancement. Urinary bladder, left terminal ureter, and entire right ureter appeared normal. Uterus showed a contraceptive device in situ, but it was otherwise normal. Both the ovaries were normal in size but showed multiple peripherally arranged follicles. The differential diagnosis on CT included fibromatosis or nodal disease due to relapsed tuberculosis. Because serum thyroglobulin was normal $(<5 \mathrm{ng} / \mathrm{mL})$, the possibility of metastasis from papillary carcinoma thyroid was considered remote. Following $\mathrm{CT}$, the patient underwent ureteroscopy with double J (DJ) stenting to relieve the ureteric obstruction. Ureteroscopy revealed no mucosal lesion and confirmed narrowing due to extrinsic compression. Urine cytology was normal.

Magnetic resonance imaging (MRI) of the pelvis was ordered to further characterize the mass. MRI was performed on GE Signa HDxt 1.5T machine. Sequences performed are described in -Table 1. On MRI, an elongated soft tissue intensity lesion showing two nodular, dumbbell-shaped components was seen medial to the left lower ureter just below the pelvic brim reaching up to the posteromedial wall of left external iliac vein. There was focal thickening of the adjacent pelvic fascia. The lesion appeared hypointense on $\mathrm{T} 1 \mathrm{~W}$ sequence. On T2W sequences, the anterior component appeared more hyperintense whereas 
Table $1 \mathrm{MRI}$ sequences performed

\begin{tabular}{|l|l|l|l|l|l|l|l|l|}
\hline Sequence & FOV & $\begin{array}{l}\text { Slice } \\
\text { thickness }\end{array}$ & Slice gap & $\begin{array}{l}\text { Matrix size } \\
\text { (frequency encoding } \\
\text { × phase encoding) }\end{array}$ & TE & TR & $\begin{array}{l}\text { Receiver } \\
\text { bandwidth }\end{array}$ & $\begin{array}{l}\text { Flip angle } \\
\text { (in degrees) }\end{array}$ \\
\hline Coronal T2 (BH) & 42 & 6 & 1 & $288 \times 224$ & 90 & 1,000 & 62 & 90 \\
\hline Axial T2 FS (RTR) & 44 & 5 & 1 & $288 \times 244$ & 90 & 1,000 & 62 & 90 \\
\hline Axial T1 (BH) & 44 & 5 & 1 & $288 \times 244$ & 5 & 180 & 62 & 90 \\
\hline Axial FIESTA (BH) & 44 & 5 & 1 & $224 \times 224$ & 1.8 & Auto $^{\text {a }}$ & 83 & 70 \\
\hline DWI (RTR) & 46 & 5 & 1 & $128 \times 140$ & 68 & Auto $^{\text {a }}$ & 250 & $\begin{array}{l}\text { Diffusion gradi- } \\
\text { ent applied }\end{array}$ \\
\hline
\end{tabular}

Abbreviations: BH, breath hold; DWI, diffusion-weighted imaging; FIESTA, fast imaging employing steady-state acquisition; FOV, field of view; MRI, magnetic resonance imaging; RTR, respiratory triggered; TE, echo time; TR, repetition time.

${ }^{a}$ Autocalculated by machine depending on number of slices.

the posterior component was of dark signal (-Fig. 2). There was no evidence of diffusion restriction. The lesion was distinct from the left ovary, fallopian tube, and uterus. Contrast-enhanced sequences were not performed. The differential diagnosis on MRI included nodal disease due to relapsed tuberculosis, retroperitoneal fibrosis(RPF), desmoid tumor, deep pelvic endometriosis, and amyloidosis with a remote possibility of metastasis from thyroid primary.

Positron emission tomography-computed tomography (PET-CT) was ordered to look for more easily accessible sites for biopsy. PET-CT was performed on Philips Gemini TOF 16 machine after intravenous administration of radiolabeled glucose 2-deoxy-2-( $\left.{ }^{18} \mathrm{~F}\right)$ fluoro-D-glucose (FDG) (in a dose of 3-5 MBq/ $/ \mathrm{kg}$ ) and scan obtained 1 hour after the injection of radioactive FDG. PET-CT scan showed increased uptake in both the nodular components of the mass with SUV of 7-8 ( - Fig. 3 ). There was no other evidence of active disease elsewhere, including in the thyroid bed or neck nodes. CT-guided fineneedle aspiration cytology (FNAC) was performed instead of biopsy due to the proximity of the mass to the vessels.

Aspiration cytology material was scanty. The pathologist noted three sheets of glandular epithelial cells. These showed bland nuclei with pale chromatin and occasional intranuclear grooves. These glandular epithelial cells did not appear to be mesothelial cells or carcinoma cells. However, few scattered stromal cells were noted outside the sheets $(-$ Fig. 4$)$. The pathologist considered the possibility of endometriosis in this situation. Because extra material was not available, FNAC smears were destained and immunocytochemistry (ICC) was performed to confirm the endometrial origin. On ICC the epithelial cells showed positivity for ER, PAX8, and WT1 and negative for TTF1 (more markers could not be asked for). Mullerian origin was confirmed on ICC, and diagnosis of ureteric endometriosis was favored by the pathologist in this female patient of reproductive age group presenting with extraureteric compression.

After the pathologist favored the diagnosis of endometriosis, a multidisciplinary meeting was convened involving the treating surgical oncologist, uro-oncologist, gyne-oncologist, radiologist, and pathologist. The imaging findings were reviewed carefully, and a final preoperative radiopathologic diagnosis of deep pelvic endometriosis causing extrinsic ureteral compression was considered, although the patient did not have a typical history that suggested endometriosis and her gynecologist did not concur with this diagnosis. The patient underwent surgical excision of the lesion at a general hospital, and the postoperative histopathologic examination showed findings suggestive of endometriosis.

\section{Discussion}

Papillary thyroid cancer is the most common welldifferentiated thyroid cancer contributing to $85 \%$ of all thyroid malignancies. ${ }^{1}$ Papillary thyroid carcinoma tends to be
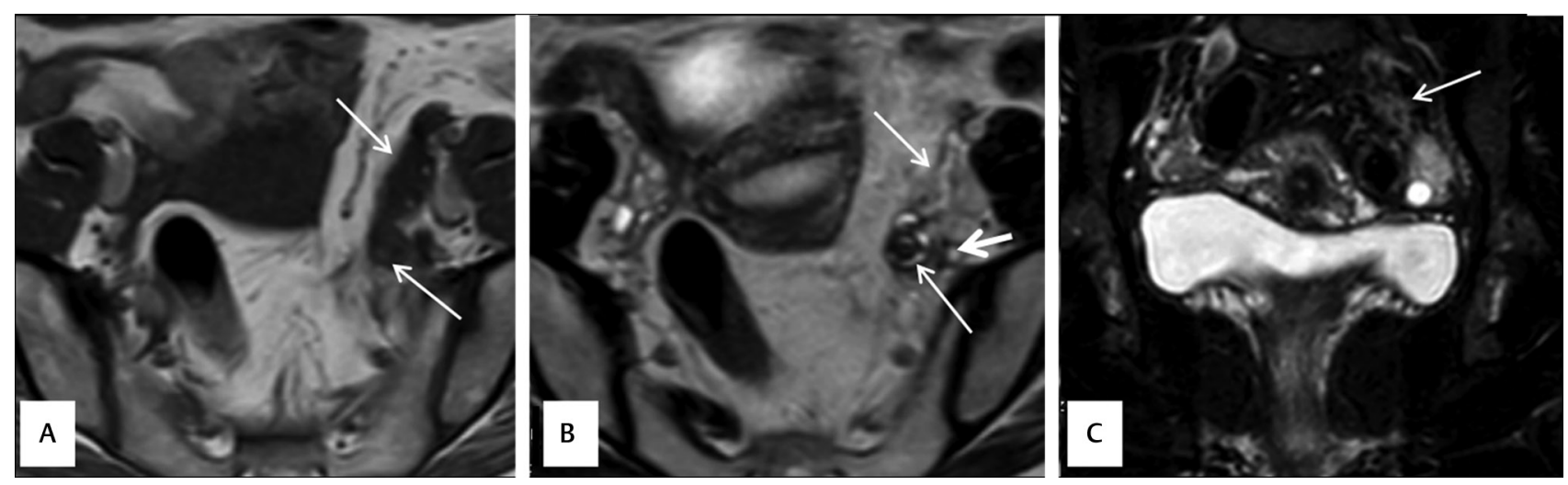

Fig. 2 Axial T1W (A), axial T2W (B), and coronal STIR (C) MRI scans show an elongated mass that is T1 hypointense and T2 heterogeneous (long arrows in $\mathbf{A}$ and $\mathbf{B}$ ), compressing the left ureter (thick arrow in B). Mass is hyperintense on STIR but less than the ovary (arrow in $\mathbf{C}$ ). 


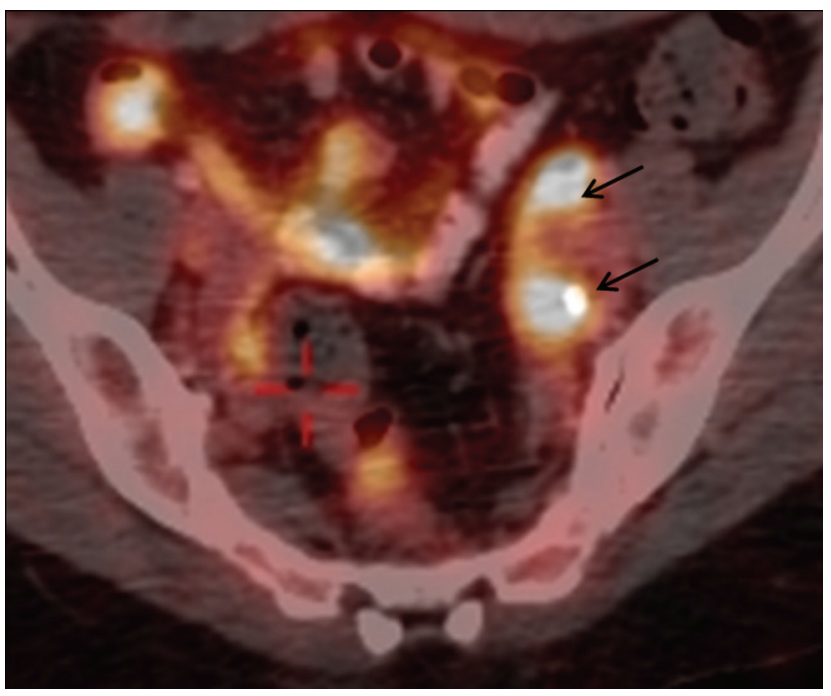

Fig. 3 A Positron emission tomography-computed tomography (PET-CT) scan shows increased uptake in the dumbbell-shaped components of the left periureteral mass (arrows).

multifocal and metastasizes early to the neck nodes. ${ }^{2}$ Bloodborne metastasis to distant sites is rare. Known sites of distant spread in papillary thyroid carcinoma include the bone, lung, skin, and brain whereas involvement of recurrent laryngeal nerve, larynx, pharynx, trachea, and esophagus occurs due to direct infiltration by the tumor. ${ }^{3}$ There are also reports of distant metastases to the kidney, ${ }^{4}$ cerebellum, ${ }^{5}$ skin, ${ }^{6}$ and esophagus ${ }^{7}$ in the literature. Though there are many different staging systems, the American Thyroid Association guidelines recommend use of the Union for International Cancer Control/American Joint Committee on Cancer (UICC/AJCC) TNM staging system for differentiated thyroid carcinoma. ${ }^{8}$ Overall 10 -year survival rate of locoregional papillary thyroid cancer is greater than $95 \%{ }^{9}$ Therefore, the treatment for locoregional disease includes total thyroidectomy and neck node dissection, followed by ablation of the residual thyroid tissue by the $\mathrm{I}^{131}$ radio iodine.

Serum thyroglobulin level is used as a marker for recurrent or residual differentiated thyroid cancer with a reference level of less than $5 \mathrm{ng} / \mathrm{mL} .{ }^{10}$ It is secreted by the normal thyroid cells as well as tumor cells. Therefore, in patients with total thyroidectomy, serum thyroglobulin level can be a useful marker to look for residual, recurrent, or metastatic disease. When increased, neck ultrasonogram and ${ }^{131}$ I or ${ }^{123}$ I wholebody scans (WBS) are obtained. Some of the differentiated thyroid cancers may undergo de-differentiation. ${ }^{11,12}$ They may not secrete thyroglobulin, and ${ }^{131} \mathrm{I}$ or ${ }^{123}$ I scan may be negative. In this case, FDG PET-CT scan is important to rule out recurrent or metastatic disease. ${ }^{13}$

Poor prognostic factors for papillary thyroid cancer include age more than 55 years, nodal or distant metastasis, extrathyroid tumor extension, and large tumor size at presentation. ${ }^{14}$ This patient was stage I (T1NOM0) at the time of surgery with complete surgical excision and also patient was far younger than 55 years.

Stage I (T1NOM0) disease at presentation with absence of poor prognostic factors, normal thyroglobulin level, and serial negative ultrasound did not favor a diagnosis of thyroid metastatic disease based on CT scan. PET-CT showed increased uptake in the periureteral lesion, but because of the normal thyroglobulin level and absence of poor prognostic indicators for papillary thyroid cancer at the time of presentation, thyroid metastasis was still considered unlikely. A relapse of tuberculosis was considered more likely.

On CT and MRI scans, the mass was periureteral, extrinsic, and ill-defined causing ureteral compression and situated deep in the pelvis. Therefore, our differentials included entities such as amyloidosis, RPF, tuberculosis, desmoid tumor, and endometriosis.

Amyloidosis can rarely involve the ureter, causing focal or diffuse wall thickening with or without periureteral stranding, ureteral filling defect, or focal masses. ${ }^{15,16}$ It does not involve the ureter circumferentially. On imaging, amyloidosis can be confused with malignancy and infection. Therefore, diagnosis is mainly on histopathology with characteristic apple-green birefringence under a polarizing microscope. ${ }^{16}$ In this case, lesion was extrinsic, but with no features of amyloidosis on histopathology.

RPF is characterized by development of fibrous plaque in the retroperitoneum often encasing the ureter and causing obstruction. RPF can be active or chronic. Active RPF shows high T2 signal intensity and postcontrast enhancement and

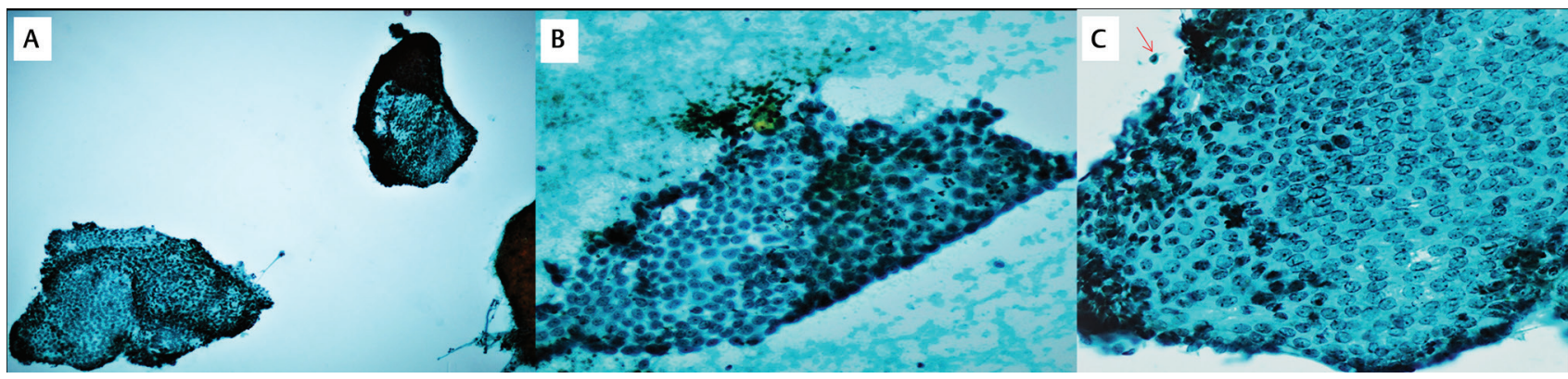

Fig. 4 (A) A fine-needle aspiration cytology (FNAC) smear showing two sheets of glandular epithelial cells. Note the background is clear Pap smear (100×). (B) Smear shows a folded honeycombed sheet of epithelial cells. Few stromal cells are noted in the background (200x). (C) Higher magnification reveals crowded glandular epithelial cells showing uniform oval pale nuclei with micronucleoli and occasional intranuclear groove. Also note a stray stromal cell outside the epithelial sheet (arrow) (400×). 
PET uptake. Chronic RPF shows low T2 signal intensity with no postcontrast enhancement and no PET uptake. ${ }^{17,18}$ Also, RPF pulls the ureter medially. In this case, the lesion was T2 dark, but it showed uptake on PET-CT scan and there was no medial deviation of the ureter.

This patient had history of tuberculosis in the past. Therefore, relapse of tuberculosis was a likely diagnosis. Primary tuberculosis of the ureter causes intrinsic involvement of the ureter with irregular, ragged wall thickening, filling defect, obstruction, and periureteric inflammatory changes. Chronic fibrotic stricture causes beaded or corkscrew appearance. Chronic wall thickening may also cause shortening of the ureter (pipestem ureter). ${ }^{19,20}$ In this patient, lesion was extrinsic with no intrinsic involvement of the ureteric wall with no shortening, ruling out primary ureteral tuberculosis. There was possibility of tuberculous nodal involvement that could cause extrinsic compression of the ureter. However, the mass was located medial to the ureter that is an unusual site for nodes. However, it was included in the list of our differentials because of the prevalence in India, past history of tuberculosis, and the imaging features. However, on histopathology, absence of granuloma or caseous necrosis ruled out tuberculosis.

Desmoid tumor belongs to the group of disorder called fibromatosis, which are characterized by the fibroblastic proliferation without any evidence of inflammation or neoplasia. Fibromatosis can be superficial or deep. Desmoid tumor belongs to the category of deep fibromatosis (locally aggressive) and constitutes less than $1 \%$ of all retroperitoneal tumors. They can be sporadic or familial and associated with familial adenomatous polyposis or Gardner's syndrome. Desmoid tumor is also responsive to estrogen and is more common in women in the reproductive age group (10-40 years). Imaging appearances of the desmoid tumors depend on tumor composition (spindle cells, collagen, myxoid matrix). Early tumors have more myxoid component with increasing collagen deposition with advancing stage. They can be well defined or ill defined and have mass effect on the surrounding organs, for example the ureter, bowel, etc. On CT scan, they show variable density depending on the composition, hypodense in early tumors becoming hyperdense in late stage with increasing collagen deposition, and may show moderate to intense contrast enhancement. On MRI, early cellular desmoid tumors with more myxoid content appear hyperintense on T2WI, becoming T2 hypointense in late stage with low cellularity and increasing collagen deposition. ${ }^{21,22}$ This patient was in reproductive age group and also had imaging findings of desmoid tumor as discussed previously; hence, it was one of our differentials.

Endometriosis is a rare cause of periureteric mass causing proximal hydroureteronephrosis. ${ }^{23,24}$ It is seen in women in the reproductive age group. Solid endometriosis, which is also referred to as deep pelvic endometriosis or deeply infiltrative endometriosis, is defined by the extension of endometrial glands and stroma at least $5 \mathrm{~mm}$ beneath the peritoneal surface. ${ }^{25}$ Unlike endometriomas, which contain viscous proteinaceous and hemorrhagic contents, solid masses of endometriosis are composed of ectopic endometrial gland and stromal cells embedded within dense fibrous tissue and smooth muscle. Also, they are usually located below the pelvic brim. On MRI, relatively acellular regions of fibrous tissue and compact smooth muscle have intermediate signal intensity on T1-weighted MRI scans and low signal intensity on T2-weighted images. On T2-weighted images, solid endometriotic masses or nodules will appear as hypointense masses with irregular, indistinct, or stellate margins due to the presence of abundant fibrous tissue and smooth muscle proliferation. Areas of T2 hyperintensity can be seen within, which likely represent endometrial glands. ${ }^{26,27}$ Adjacent organ invasion (bladder, rectum, or ureter) may also be seen. ${ }^{26}$ Although the menstrual history did not favor this diagnosis, these findings were seen in this patient, which prompted the authors to favor this differential diagnosis upon reviewing imaging features at the multidisciplinary meeting. Endometriosis was confirmed on postoperative histopathologic examination.

Endometriosis is the presence of endometrial gland and/or stroma outside the endometrial cavity. It is more commonly seen in infertile and nulliparous women. The prevalence of ureteral endometriosis ranges from 0.01 to $1 \%$ of all women with disease. ${ }^{28}$ Ureteral endometriosis can be intrinsic or extrinsic. Extrinsic endometriosis involves the adventitia of the ureter and surrounding tissue whereas intrinsic ureteral endometriosis involves the muscularis, lamina propria, or lumen of the ureter, either from the deep infiltrating periureteral lesion or directly from the lymphatic or hematogenous metastasis of the endometrial tissue. ${ }^{26}$ Extrinsic involvement of the ureter (80\%) is more common than the intrinsic involvement (20\%). ${ }^{29}$

The etiology and pathogenesis of endometriosis is still unclear. Several theories have been proposed for etiopathogenesis of endometriosis. Broadly, these are the theories that propose that implants (1) originate from uterine endometrium or (2) arise from tissues other than the uterus. These include metaplastic theory, induction theory, theory of embryonic mullerian rests, extrauterine stem or progenitor cell theory, benign metastatic theory, and theory of retrograde menstruation. ${ }^{26,30}$ According to retrograde menstruation theory, viable endometrial tissue refluxes through the fallopian tube and gets deposited in the pelvic organs. This latter theory is favored by the anatomic distribution of endometriotic lesions. Superficial implants are more often located in the posterior compartment of the pelvis and in the left hemipelvis. ${ }^{31}$ The terminal part of the lower ureter is first related to the posterior border of the ovary and then lies in the uterosacral ligament, both being common sites for endometriosis. In this case, the implant was on the left side and posteriorly located involving the terminal lower ureter. However, surprisingly the patient had no history suggesting endometriosis. She had also completed her family 10 years before and was asymptomatic since then.

\section{Conclusion}

The occurrence of a second benign lesion baffling the clinician in a treated case of cancer is not an infrequent phenomenon 
in a referral cancer center. This case highlights the challenge of confidently diagnosing deep pelvic endometriosis in an asymptomatic 41-year-old multiparous woman who was previously treated for both papillary thyroid cancer (PTC) and abdominal tuberculosis. Well-differentiated papillary carcinomas of the thyroid in patients younger than 55 years, with absence of nodal or systemic metastasis, lymphovascular invasion, extrathyroid spread, and small size $(<5 \mathrm{~cm})$, are considered low risk. These rarely metastasize to distant organs, and this fact should be kept in mind, particularly when the thyroglobulin is within normal limits. The differential diagnoses for periureteral pelvic peritoneal lesions has been discussed, and the imaging features of deep pelvic endometriosis has been described. Endometriosis should always be included in the differential diagnosis of a pelvic mass in women of childbearing age group.

\section{Conflict of Interest}

None declared.

\section{References}

1 National Cancer Institute. Surveillance Epidemiology and End Results Data 1983 to 2009

2 Cotran RD, Kumar VCT. Robbins Pathological Basis of Disease. 6th ed. Philadelphia, PA: WB Saunders; 1999:1143-1144

3 McCaffrey TV, Bergstralh EJHI, Hay ID. Locally invasive papillary thyroid carcinoma: 1940-1990. Head Neck 1994;16(2):165-172

4 Graham LDRS, Roe SM. Metastatic papillary thyroid carcinoma presenting as a primary renal neoplasm. Am Surg 1995;61(8):732-734

5 Pacak K, Sweeney DC, Wartofsky L, et al. Solitary cerebellar metastasis from papillary thyroid carcinoma: a case report. Thyroid 1998;8(4):327-335

6 Lissak B, Vannetzel JM, Gallouedec N, Berrod JLRM, Rieu M. Solitary skin metastasis as the presenting feature of differentiated thyroid microcarcinoma: report of two cases. J Endocrinol Invest 1995;18(10):813-816

7 Cooney BS, Levine MSSM, Schnall MD. Metastatic thyroid carcinoma presenting as an expansile intraluminal esophageal mass. Abdom Imaging 1995;20(1):20-22

8 Edge SE, Byrd DR, Compton CC, Fritz AG, Greene FL. TAI. AJCC Cancer Staging Manual. 7th ed. New York, NY: Springer; 2010

9 Hundahl SA, Fleming ID, Fremgen AMMH, Menck HR. A National Cancer Data Base report on 53,856 cases of thyroid carcinoma treated in the U.S., 1985-1995 [see comments]. Cancer 1998;83(12):2638-2648

10 Franceschi M, Kusić Z, Franceschi D, Lukinac L, Roncević S. Thyroglobulin determination, neck ultrasonography and iodine-131 whole-body scintigraphy in differentiated thyroid carcinoma. J Nucl Med 1996;37(3):446-451

11 Park JH, Kwon HJ, Park CS, Hong S. Anaplastic transformation of papillary thyroid carcinoma in a young man: a case study with immunohistochemical and BRAF analysis. Korean J Pathol 2014;48(3):234-240
12 Barbu CG, Florin A, Neamțu MC, et al. Papillary thyroid carcinoma with anaplastic dedifferentiation in the lymph node metastasis-a rare form of presentation even for a tall cell variant. Rom J Morphol Embryol 2015;56(2):527-531

13 Marcus C, Whitworth PW, Surasi DS, Pai SI, Subramaniam RM. PET/CT in the management of thyroid cancers. AJR Am J Roentgenol 2014;202(6):1316-1329

14 Passler C, Scheuba C, Prager G, et al. Prognostic factors of papillary and follicular thyroid cancer: differences in an iodine-replete endemic goiter region. Endocr Relat Cancer 2004;11(1):131-139

15 Hayashi T, Kojima S, Sekine H, Mizuguchi K. Primary localized amyloidosis of the ureter. Int J Urol 1998;5(4):383-385

16 Weiwei Z, Yi H, Jinsong Z. Primary localized amyloidosis of the ureter. Abdom Imaging 2011;36(5):609-611

17 Wasnik AP, Elsayes KM, Kaza RK, Al-Hawary MM, Cohan RH, Francis IR. Multimodality imaging in ureteric and periureteric pathologic abnormalities. AJR Am J Roentgenol 2011;197(6):W1083-92

18 Geoghegan T, Byrne AT, Benfayed W, McAuley G, Torreggiani WC. Imaging and intervention of retroperitoneal fibrosis. Australas Radiol 2007;51(1):26-34

19 Harisinghani MG, McLoud TC, Shepard JA, Ko JP, Shroff M, Mueller PR. Tuberculosis from head to toe. Radiographics 2000;20(2):449-470, quiz 528-529, 532

20 Jung YY, Kim JK, Cho KS. Genitourinary tuberculosis: comprehensive cross-sectional imaging. AJR Am J Roentgenol 2005;184(1):143-150

21 Casillas J, Sais GJ, Greve JL, Iparraguirre MC, Morillo G. Imaging of intra- and extraabdominal desmoid tumors. Radiographics 1991;11(6):959-968

22 Rajiah P, Sinha R, Cuevas C, Dubinsky TJ, Bush WH Jr, Kolokythas O. Imaging of uncommon retroperitoneal masses. Radiographics 2011;31(4):949-976

23 Ponticelli C, Graziani G, Montanari E. Ureteral endometriosis: a rare and underdiagnosed cause of kidney dysfunction. Nephron Clin Pract 2010;114(2):c89-c93

24 Machairiotis N, Stylianaki A, Dryllis G, et al. Extrapelvic endometriosis: a rare entity or an under diagnosed condition? Diagn Pathol 2013;8:194

25 Coccia MERF, Rizzello F. Ultrasonographic staging: a new staging system for deep endometriosis. Ann N Y Acad Sci 2011;1221:61-69

26 Coutinho A Jr, Bittencourt LK, Pires CE, et al. MR imaging in deep pelvic endometriosis: a pictorial essay. Radiographics 2011;31(2):549-567

27 Siegelman ES, Oliver ER. MR imaging of endometriosis: ten imaging pearls. Radiographics 2012;32(6):1675-1691

28 Jubanyik KJCF, Comite F. Extrapelvic endometriosis. Obstet Gynecol Clin North Am 1997;24(2):411-440

29 Wang MT, Mak CW, Tzeng WS, Chen JC, Chang JM, Su CC. Radiographic features of intrinsic ureteral endometriosis: a case report. Zhonghua Fang She Xue Za Zhi 2005;30:59-63

30 Burney RO, Giudice LC. Pathogenesis and pathophysiology of endometriosis. Fertil Steril 2012;98(3):511-519

31 Al-Fozan H, Tulandi T. Left lateral predisposition of endometriosis and endometrioma. Obstet Gynecol 2003;101(1):164-166 